fetal capacity for growth and minimizes maternal ability to support it) and unlikely for later-born females with a large mother and small father (a combination which reverses the major influences). In respect of the probability of retarded growth, most normal single fetuses fall between these extremes.

An explanation of a duration of pregnancy beyond the period of maximal rate of fetal growth must presumably be sought in the advantages in natural selection of maturity at birth. It seems clear that in large mammals, including primates, selection has favoured reduction of litter size and pnolongation of pregnancy. And even when there is only one fetus it may still be an advantage for it to be retained in the uterus if in consequence it is better fitted to meet the risks of death encountered after birth. At least during the later part of man's evolution the risks from infectious disease were formidable, and the development of immunity may have been among the most important reasons for prolongation of gestation length. There is little doubt that though the child is not mature in the sense that the hare and foal are mature at birth, as judged by ability to care for themselves in the postnatal environment, nevertheless it is somewhat better equipped immunologically at $\mathbf{4 0}$ than at 36 weeks.

The evidence discussed above suggests that the retardation of fetal growth in single pregnancy, and even the more pronounced retardation in twin pregnancy, have little influence on intelligence as measured in the 11-plus examination. This conclusion is based on the very small differences in verbal reasoning scores between sibs of different weights and durations of gestation, and still more convincingly on the observation that twins whose co-twins do not survive beyond one month after birth perform almost as well as single births, whereas when both twins survive the results are substantially lower (Record et al., 1970). This finding overcomes many difficulties in interpretation, and since variation due to birth weight and duration of gestation, maternal age and birth rank, type of twinning and order of birth can be eliminated, it can hardly be explained except as a result of differences in postnatal experience between single and paired twins.

The data also threw light on the prolonged debate concerning the interpretation of the relation between measured intelligence and family size, which led to the conclusion that intelligence was declining. This relation is due mainly to a difference between rather than within families and cannot be dismissed as a result of a familial association between intelligence and birth rank. Nevertheless, all the evidence, including particularly the experience of single and paired twins, points to a postnatal rather than a congenital explanation for the observed variation.

REFERENCES

Barker, D. J. P. (1966). British Fournal of Preventive and Social Medicine, 20, 58.

Drillien, C. M. (1964). The Growth and Development of the Prematurely Born Infant. Livingstone, Edinburgh.

Leitch, I., Hytten, F. E., and Billewicz, W. Z. (1959). Proceedings of the Zoological Society, 113, 11.

McKeown, T., and Record, R. G. (1952). Fournal of Endocrinology, 8, 386.

Mckeown, T., and Record, R. G. (1953). Fournal of Endocrinology, 9, 418.

Record, R. G., McKeown, T., and Edwards, J. H. (1969a). Annals of Human Genetics, 33, 61.

Record, R. G., McKeown, T., and Edwards, J. H. (1969b). Annals of Human Genetics, 33, 71.

Record, R. G., McKeown, T., and Edwards, J. H. (1970). Annals of Human Genetics. In press.

\title{
Diagnosis of Pulmonary Embolism. A Correlative Study of the Clinical, Scan, and Angiographic Findings
}

\author{
KATTADIYIL P. POULOSE, ${ }^{*}$ M.D. ; RICHARD C. REBA,† M.D. ; DAVID L. GILDAY, $\ddagger$ M.D. \\ FRANK. H. DELAND, $\subseteq$ M.D. ; HENRY N. WAGNER, JUN.,\| M.D.
}

\begin{abstract}
Summary: Seventy-one patients with suspected pul$\checkmark$ monary embolism had both pulmonary scans and angiograms. Clinically it was impossible to predict the absence or presence of subsequent angiographic evidence of pulmonary embolism in the individual patient. In 24 patients the scans showed defects that were believed to indicate a high probability of pulmonary embolismnamely, perfusion defects that corresponded to specific anatomical segments of the lung together with normal chest radiographs or radiographs suggestive of pulmonary embolism. Specific angiographic evidence of pulmonary embolism was found in $18(75 \%)$ of these patients. Diffuse, patchy, and non-segmental perfusion defects were seen in 28 patients. Only seven $(25 \%)$ of this group had angiographic abnormalities specific for embolism. In 12 patients with normal lung scans none showed subsequent
\end{abstract}

* Instructor, Division of Nuclear Medicine, Johns Hopkins Medical Institutions, Baltimore, Maryland, U.S.A.

† Chairman, Division of Nuclear Medicine, Washington Hospital Center, Washington, D.C.

‡ Department of Radiology, Winnipeg General Hospital, Winnipeg, Canada.

$\$$ Associate Professor, Division of Nuclear Medicine, Johns Hopkins Medical Institutions, Baltimore, Maryland, U.S.A.

| Professor, Division of Nuclear Medicine, Johns Hopkins Medical Institutions, Baltimore, Maryland, U.S.A. evidence of embolism by angiography. It is concluded firstily that lung scanning is a sensitive screening procedure for pulmonary embolism and, secondly, that segmental perfusion defects are highly suggestive of pulmonary embolism.

\section{Introduction}

Pulmonary embolism is an exceedingly common and often lethal disease (Soloff and Rodman, 1967). In unselected necropsy cases an incidence of $51-64 \%$ was reported by several authors (Freiman et al., 1965; Morrell and Dunnill, 1968). The symptoms of massive pulmonary embolism are acute, severe, and very striking, but in many patients symptoms may be slight or even absent and diagnosis is difficult. When pulmonary embolism is not immediately fatal, spontaneous resolution is the rule rather than the exception, so that most patients recover. The correct diagnosis of pulmonary embolism is becoming important because of the effectiveness of anticoagulant therapy and the potential effectiveness of the new thrombolytic agents, such as urokinase and streptokinase.

Two relatively recent diagnostic procedures-selective pulmonary arteriography and lung scanning-have assumed progressively increasing importance in the management of patients suspected of pulmonary embolism. 


\section{Patients and Methods}

Seventy-one patients admitted to the Johns Hopkins Hospital, in whom pulmonary embolism was thought to be the most probable clinical diagnosis and who subsequently had lung scan followed by pulmonary angiogram, form the basis of this report. The interval between the scan and the angiogram varied from two hours to three days in $80 \%$ of the cases and in the remainder within three to five days. The clinical symptoms and signs, coexistent diseases, and pertinent laboratory data in these 71 patients were tabulated and analysed.

Lung scans were performed with a rectilinear scanner after the intravenous administration of the radiopharmaceutical. At least two views-posterior and lateral-were obtained in
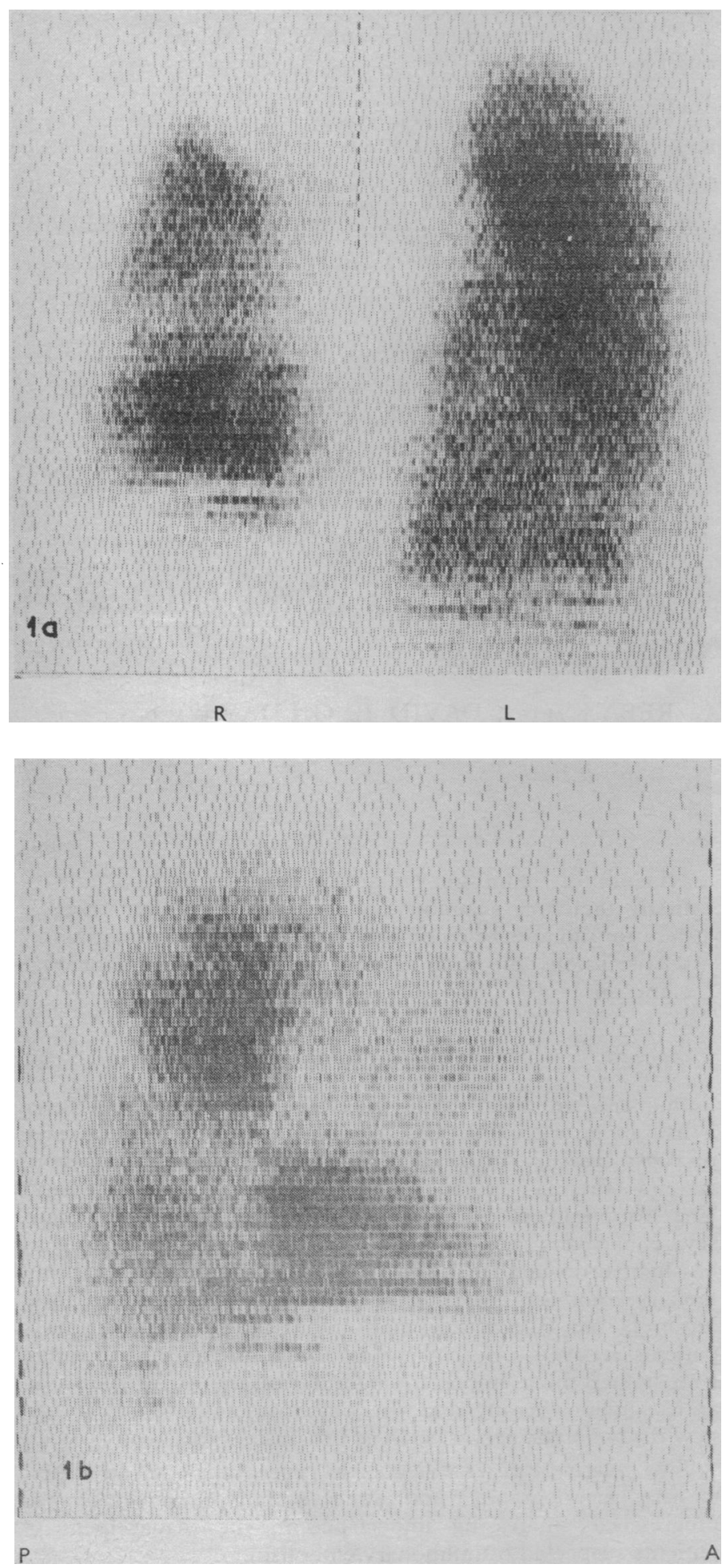
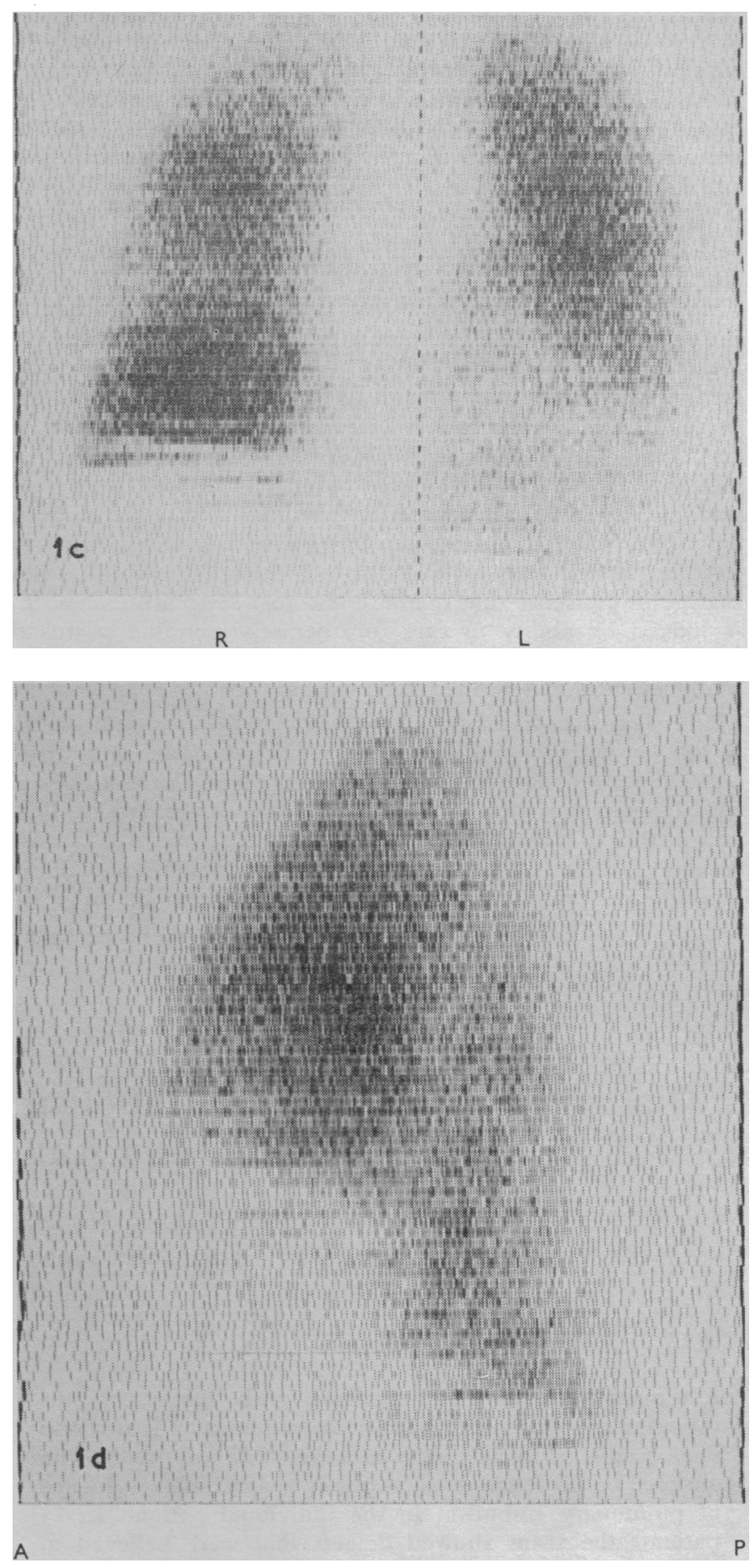

Fig. 1.-Case 1092464. Lung scans (a) Posterior view, showing diminFIG. 1.-Case 1092464. Lung scans (a) Posterior view, showing dimin-
ished perfusion in the right midlung field with a peripheral concave ished perfusion in the right midlung field with a peripheral concave (b) Right lateral view, showing the perfusion defects corresponding to (b) Right lateral view, showing the perfusion defects corresponding to the anterior segment of the upper lobe, lateral segment of the middle lobe, and apical and lateral basal segments of the lower lobe. (c)
Repeat scan after 12 days. Posterior view showing two perfusion defects in the right lung field and one in the left lower lung field. When compared with the first scan (a), note the improved perfusion towards the upper and lower lobes of the right lung and less perfusion towards the left lower lung field. (d) Left lateral view, showing an oblique linear area of diminished perfusion in the region of the major fissure, as well as involvement of the whole left lower lobe, the posterior basal segment being affected more. $\mathrm{A}=$ Anterior. $\mathrm{P}=$ Posterior.

Scans with this type of segmental perfusion defects are interpreted as having a "high probability for pulmonary embolism." Pulmonary arteriography in this patient showed multiple pulmonary emboli.

every instance, and whenever possible all four viewsanterior, posterior, and both laterals-were obtained. The radiopharmaceuticals used were ${ }^{99 m} \mathrm{Tc}$ albumin microspheres, 
${ }^{113 m}$ InFe (OH), or ${ }^{131} \mathrm{I}$-MAA. The scans were interpreted independently by two observers without knowledge of the angiographic findings and classified into four groups: (1) high probability of pulmonary embolism: perfusion defects (diminished or absent perfusion) segmental in distribution accompanied by a normal chest radiograph or by radiographic signs known to occur in pulmonary embolism, and without clinical evidence of bronchospasm (Fig. 1); (2) medium probability of pulmonary embolism: perfusion defects diffuse in nature not confined to any pulmonary segments, or defects with segmental and non-segmental distribution in patients with evidence of chronic obstructive pulmonary diseases (Fig. 2); (3) low probability of pulmonary embolism: defects small and patchy in nature, or defects in the corresponding area of abnormality seen in the chest radiograph; and (4) normal lung scans. In the last group the clinical suspicion was high enough for a pulmonary angiogram to be taken despite a normal lung scan.

$2 a$

$\mathbf{R}$
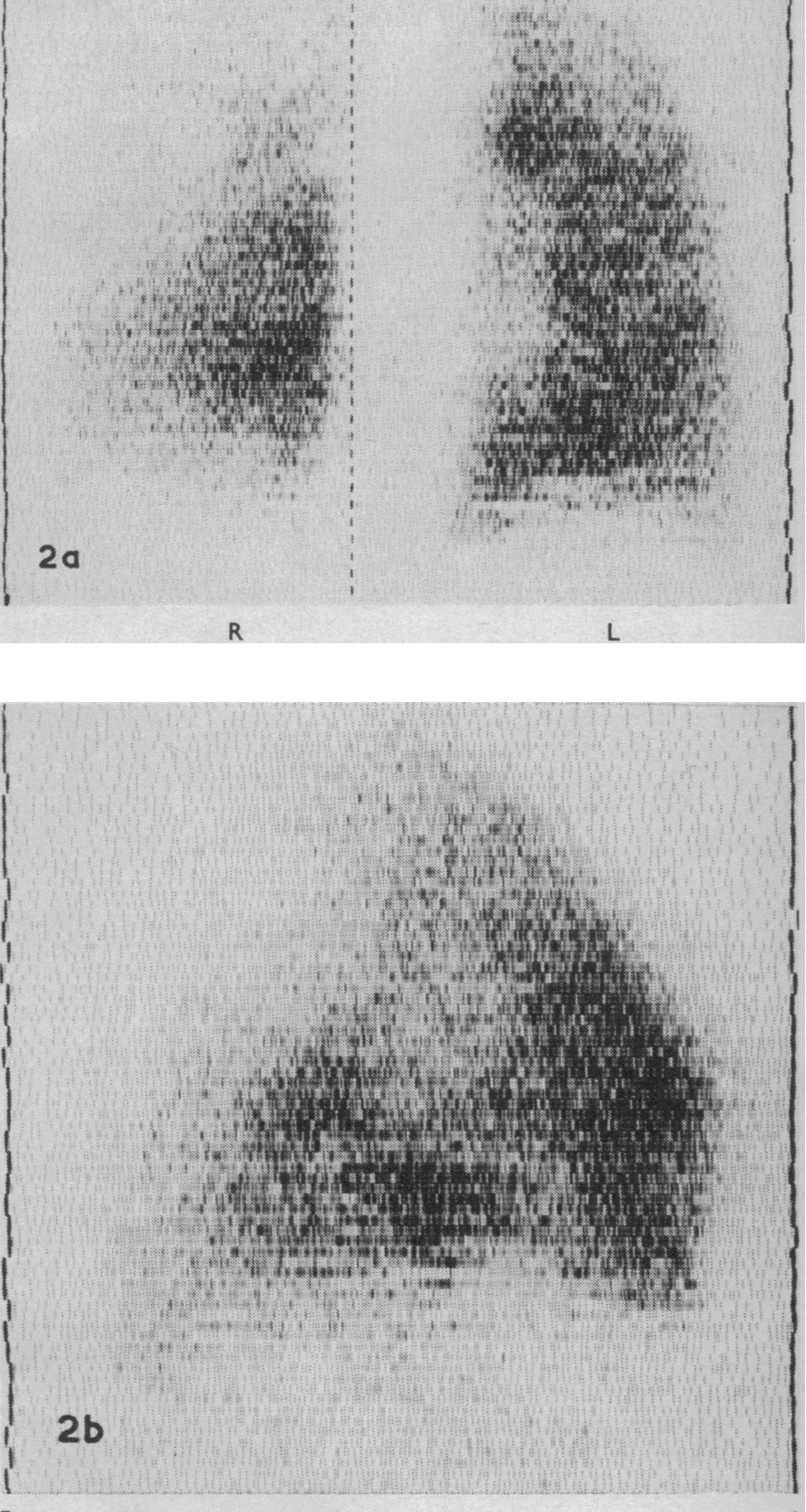

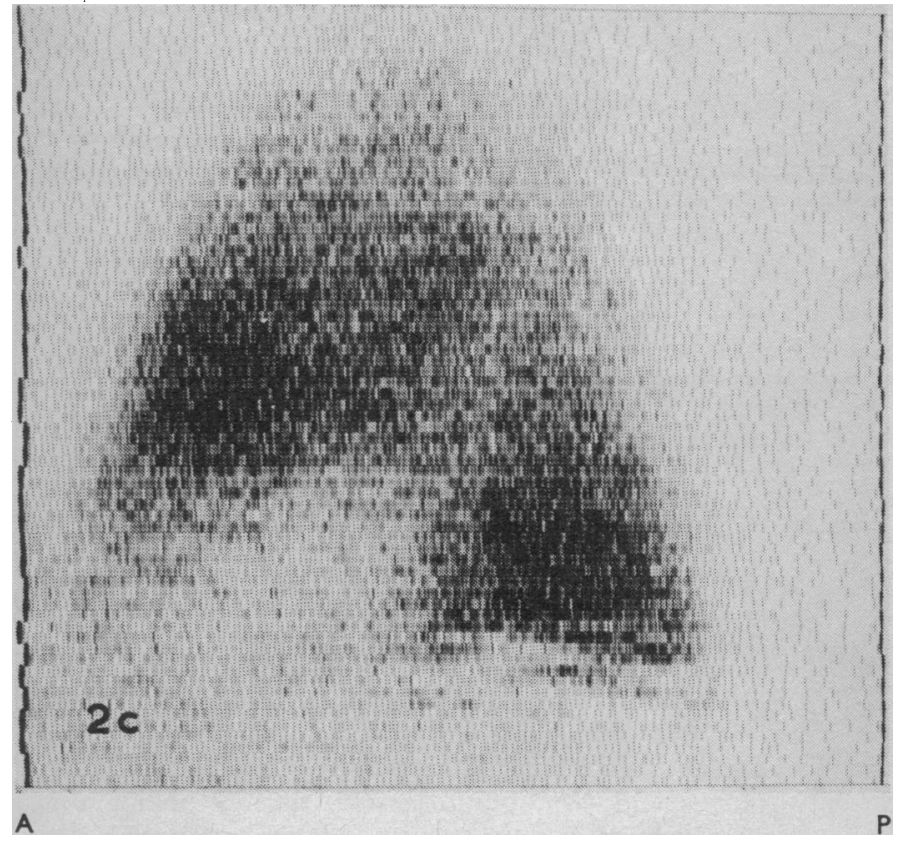

FIG. 2.-Case 457676. Lung scans. (a) Posterior view, showing diffuse involvement of the right lung field and patchy involvement of the left upper and midlung fields. (b) Right and (c) left lateral views, showing the diffuse non-segmental nature of the perfusion defects, predominantly in the upper portions. The indentation seen in the anteroinferior portion in the left lateral view is due to cardiac impression.

Pulmonary function studies in this patient showed evidence of chronic obstructive pulmonary disease. This scan is interpreted as having a "medium probability of pulmonary embolism." A pulmonary angiogram showed non-specific abnormalities compatible with chronic obstructive showed non-specific
pulmonary disease.

Pulmonary angiography was performed with a catheter in the main pulmonary artery or, in rare instances, in the right ventricle. After the rapid injection of $50-75 \mathrm{ml}$. of diatrizoic acid (Hypaque) by pressure injector, serial exposures in the anteroposterior projection were obtained. The angiograms were classified into three categories as described by Stein et al. (1967): (1) specific evidence of pulmonary embolism: definite intravascular filling defects or amputation or absence of one or more segmental or subsegmental vessels; (2) non-specific evidence: narrowing of vessels, or delayed or asymmetrical filling; and (3) normal vascular pattern.

The various types of angiographic findings in the different groups of patients classified on the basis of clinical as well as scan findings were then analysed to determine if there was any correlation between the three diagnostic modalitiesclinical, scan, and angiographic.

\section{Results}

There were 28 patients (40\%). with angiographic changes specific for pulmonary embolism, $18(25 \%)$ with non-specific angiographic changes, and $25(35 \%)$ who had a normal pulmonary arteriogram. The frequency of the various clinical symptoms and signs in each of the arteriographic groups is presented in Table I.

Symptoms.-In the patients who had angiographic changes specific for pulmonary embolism, shortness of breath was the most frequent symptom, and was seen in $70 \%$ of the cases. Other major symptoms seen in this group were fever above $100^{\circ}$ F. $\left(37.8^{\circ}\right.$ C.) $(50 \%)$, pleuritic chest pain $(42 \%)$, cough $(42 \%)$, and haemoptysis (14\%). When the incidence of the various symptoms in patients with angiographic evidence of pulmonary embolism is compared with their incidence in the other two angiographic groups, no specific symptoms clinically helpful in the diagnosis of pulmonary embolism were obser- 
TABle I.-Frequency of the Prominent Clinical Features in the Three Groups of Patients Classified on the Basis of Angiographic Findings

\begin{tabular}{|c|c|c|c|c|c|c|c|c|c|c|c|c|c|c|c|c|c|c|c|}
\hline & 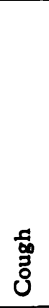 & 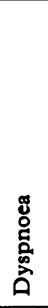 & 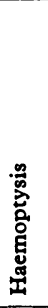 & 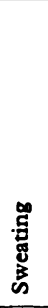 & 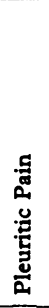 & 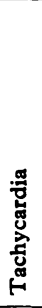 & 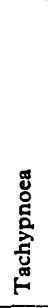 & 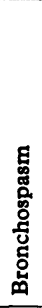 & 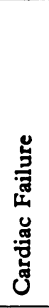 & 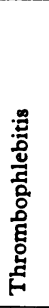 & 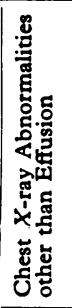 & 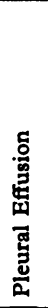 & 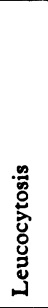 & 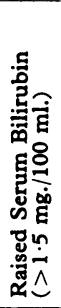 & 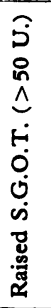 & 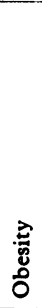 & 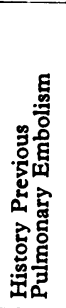 & 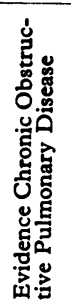 & 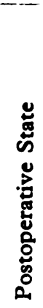 \\
\hline $\begin{array}{l}\text { Patients with specific findings } \\
\text { for pulmonary emboli }(\%)\end{array}$ & 42 & 70 & 14 & 14 & 42 & 53 & 37 & 11 & 41 & 33 & 23 & 17 & 14 & 7 & 17 & 10 & 24 & 30 & 27 \\
\hline $\begin{array}{l}\text { Patients with non-specific } \\
\text { angiographic findings }(\%)\end{array}$ & 30 & 90 & 18 & 24 & 35 & 65 & 60 & 16 & 60 & 13 & 30 & 30 & 18 & 24 & 30 & 23 & 42 & 42 & 5 \\
\hline $\begin{array}{l}\text { Patients with normal angio- } \\
\text { gram }(\%) . .\end{array}$ & 18 & 56 & 18 & 16 & 48 & 44 & 44 & 16 & 44 & 24 & 44 & 20 & 8 & 4 & 25 & 28 & 20 & 20 & 12 \\
\hline
\end{tabular}

ved. For example, shortness of breath was present in $90 \%$ of patients with non-specific angiographic findings and in $56 \%$ of those with a normal pulmonary angiogram. Pleuritic chest pain was present in $35 \%$ of patients with non-specific angiographic changes and $48 \%$ of those with normal angiograms. From the analysis of the symptoms it was not possible to predict with any degree of certainty what angiographic changes, if any, would be present in an individual patient.

Physical and Radiographic Signs.-Tachycardia (greater than $90 / \mathrm{min}$.) was present in $53 \%$ and tachypnoea (greater than $24 / \mathrm{min}$.) in $37 \%$ of patients with specific angiographic changes. The frequency of other signs is also given in Table I. Radiological abnormalities such as diminished vascular markings, linear densities, or consolidation suggestive of infarcts were seen in $23 \%$, and pleural effusion was seen in $17 \%$ of the patients in the group with specific arteriographic findings. Cardiac arrhythmias, such as atrial fibrillation, atrial flutter, supraventricular or ventricular tachycardias, and various types of heart block were present in $27 \%$ of these patients. Electrocardiographic findings suggestive of right ventricular hypertrophy, right ventricular strain, or nonspecific T-wave abnormalities were seen in $20 \%$ of the patients with angiographic evidence of pulmonary embolism. The incidence of the various signs and pertinent laboratory data in patients found to have non-specific or normal pulmonary angiograms is also given in Table I for comparison.

Predisposing Factors.-The frequency of the various coexistent conditions predisposing to pulmonary embolism in these groups of patients is also presented (Table I). These include congestive heart failure, obesity, thrombophlebitis, chronic obstructive pulmonary disease, recent major surgery (within two weeks), or orthopaedic immobilization procedures. Cardiac failure was present in $41 \%$ and thrombophlebitis in the lower extremities was clinically detected in one-third of the patients with angiographic changes specific for pulmonary embolism; however, the same features were found in $44 \%$ and $24 \%$ of patients with normal angiograms. Cardiac failure appeared much more often $(60 \%)$ in patients with nonspecific angiographic changes. We are not sure whether the cardiac failure occurred before or after the onset of symptoms which suggested pulmonary embolism. This incidence represents the number of patients who had cardiac failure at the time when pulmonary embolism was clinically suspected.

Correlation Between the Scan and Angiographic Findings.Technically satisfactory scans were obtained in only 64 patients. The frequency of specific and non-specific angiographic findings in the groups of patients classified with respect to the probability of pulmonary embolism on the basis of perfusion defects in the lung scan images is given in Table II. Seventy-five per cent. of the patients with defects confined to the various anatomical segments of the lung had angiographic findings specific for pulmonary embolism, while embolus was never found by pulmonary angiography in any TABLE II.-Patients Classified on the Basis of Scan Findings Alone
(Total Number 64)

\begin{tabular}{|c|c|c|c|c|c|}
\hline \multirow{2}{*}{\multicolumn{2}{|c|}{ Patient Group Based on Scan }} & \multirow{2}{*}{ No. } & \multicolumn{3}{|c|}{ Arteriographic Findings } \\
\hline & & & Specific & Non-specific & Normal \\
\hline $\begin{array}{l}\text { High probability (for pr } \\
\text { embolism) } \\
\text { Medium probability } \\
\text { Low probability } \\
\text { Normal scan ... }\end{array}$ & 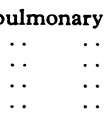 & $\begin{array}{l}24 \\
18 \\
10 \\
12\end{array}$ & $\begin{array}{l}18(75 \%) \\
4(22 \% \%) \\
3(30 \%) \\
0\end{array}$ & $\begin{array}{l}4(16 \%) \\
7(39 \%) \\
4(40 \%) \\
2(17 \%)\end{array}$ & $\begin{array}{l}2(9 \%) \\
7(39 \%) \\
3(30 \%) \\
10(83 \%)\end{array}$ \\
\hline
\end{tabular}

patient who had a normal lung scan. In the groups of patients with scans classified as "medium" and "low" probability of pulmonary embolism angiographic findings specific for emboli were observed in $22 \%$ and $30 \%$ respectively.

\section{Discussion}

The most important conclusions from this study are: (1) the arteriographic findings were correctly predicted in only $40 \%$ of the patients with high clinical suspicion of pulmonary embolism; (2) on the other hand, the lung scan correctly predicted specific arteriographic evidence of pulmonary embolism in $75 \%$ of the patients with scan defects highly suggestive of embolism; and (3) no single sign or symptom was found to occur with greater frequency in patients with specific angiographic evidence for pulmonary emboli than in those with either normal angiogram or non-specific abnormalities of the pulmonary vasculature. A similar conclusion, that there is no specific symptom or sign for pulmonary embolism, was reached by Hildner and Ormond (1967).

We have previously reported that peripheral concave perfusion defects in the posterior view were seen in $72 \%$ of patients with pulmonary embolism, and this type of defect was found more frequently in the mid-lung fields (Poulose et al., 1968). Analysis of the lateral and posterior views together showed that the concave perfusion defects seen in the periphery of the mid-lung fields are usually due to the involvement of the apical segments of the lower lobes (Fig. 1). Similarly, involvement of the posterior segment of the upper lobes or lateral basal segments of the lower lobes may also appear as peripheral concave defects in the upper or lower lung fields as seen in the posterior view. Lobar involvement may present as a diffuse type of defect in the posterior view, and the exact localization can be made only with the help of the lateral views. In rare instances the perfusion defect may be observed only in the lateral views. Seventy-five per cent. of the patients with perfusion defects corresponding to the different anatomical divisions of the lung had specific evidence of emboli in the pulmonary angiogram. By specific criteria we mean either a filling defect or amputation of segmental or larger arteries. Because of the increased frequency 
TABLE III._Clinical Suspicion of Pulmon ary Embolism. Pulmonary Perfusion Scan

\begin{tabular}{|c|c|c|c|}
\hline Scan Findings & Normal Scan & $\begin{array}{l}\text { Diffuse, Patchy, Non-segmental in } \\
\text { Distribution or Predominantly Non- } \\
\text { segmental Defects in Patients with } \\
\text { Chronic Obstructive Pulmonary Disease } \\
\text { or Bronchial Asthma }\end{array}$ & $\begin{array}{l}\text { Multiple Segmental or Subsegmental } \\
\text { Perfusion Defects with Normal Chest } \\
\text { Radiograph or with Radiographic Signs } \\
\text { known to occur in Pulmonary Embolism }\end{array}$ \\
\hline 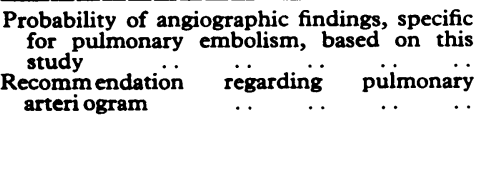 & $\begin{array}{c}\text { Very low } \\
\text { Not recommended }\end{array}$ & $\begin{array}{c}25 \% \\
\text { Recommended }\end{array}$ & $\begin{array}{l}\text { N5\% } \\
\text { Not necessary, especially in patients } \\
\text { without chronic obstructive pulmonary } \\
\text { disease, unless surgery or long-term } \\
\text { anticoagulation is contemplated }\end{array}$ \\
\hline
\end{tabular}

of segmental perfusion defects in proved cases of pulmonary embolism, we believe that any perfusion defect seen in the posterior view should be correlated with the lateral views to decide whether the defect is segmental or not. Sixteen per cent. of the patients with segmental perfusion defects had only non-specific arteriographic findings and the remaining $9 \%$ had normal arteriograms. Of the six patients in this group who had non-specific or normal arteriograms, only two had clinical evidence of chronic obstructive pulmonary disease. Probably some of the patients who did not have specific angiographic findings did in fact have pulmonary embolism. Normal or non-specific angiographic findings in proved cases of pulmonary emboli have been reported (Moser et al., 1966, Wellman et al., 1969).

In patients with chronic obstructive pulmonary disease it is unusual to find perfusion defects entirely confined to anatomical segments. These defects are usually diffuse or patchy and a superimposed embolus can never be interpreted with certainty from the scan. Diffuse or even segmental perfusion defects may occur in bronchial asthma, and pulmonary embolism can also present as asthma (Olazábal et al., 1968). Similarly, pulmonary venous hypertension secondary to congestive cardiac failure can also produce perfusion defects, usually in the bases of the lung. Thus, in patients with chronic obstructive pulmonary disease, congestive cardiac failure, or bronchial asthma only an arteriogram can establish the diagnosis. Pulmonary arteriography showed specific evidence of emboli in $25 \%$ of patients (those with "medium probability" and "low probability" scans combined) with this type of perfusion defects, while $40 \%$ had non-specific angiographic abnormalities. Non-specific angiographic abnormalities indistinguishable from pulmonary emboli occur in chronic obstructive pulmonary disease or congestive cardiac failure, thus making the angiographic interpretation also difficult. A significant number of our patients with scans grouped as "medium or low probability for pulmonary embolism" and normal or non-specific arteriograms (17 out of 21 cases) had chronic obstructive or other parenchymal disease of the lung or congestive cardiac failure.

In 12 patients the lung scans were completely normal and in none of these cases was a specific finding of emboli detected in the subsequent angiogram. We conclude that the lung scan is a simple and sensitive screening procedure for pulmonary embolism whereas the arteriogram is more specific but may be less sensitive. The diagnostic accuracy of lung scanning can be improved if (1) all the four views-anterior, posterior, and both laterals-are obtained, (2) the shape and position of the perfusion defects are anatomically localized, and (3) the scan and chest radiograph are correlated. We also recommend the diagnostic sequence shown in Table III in a patient suspected of pulmonary embolism.

Supported by U.S.P.H.S. Grant 10548.

\section{REFERENCES}

Freiman, D. G., Suyemoto, J., and Wessler, S. (1965). New England Fournal of Medicine, 272, 1278 .

Hildner, F. J., and Ormond, R. S. (1967). Fournal of the American Medical Association 202, 567.

Morrell, M. T., and Dunnill, M. S. (1968). British fournal of Surgery,

55, 347. M., Tisi, G. M., Rhodes, P. G., Landis, G. A., and Miale, A. (1966). American fournal of Cardiology, 18, 810 .

Olazábal, F., jun., Román-Irizarry, L. A., Oms, J. D., Conde, L., and Marchand, E. J. (1968). New England Fournal of Medicine, 278, 999.

Poulose. K., Reba, R. C., and Wagner, H. N., jun. (1968). New England Fournal of Medicine, 279, 1020

Soloff, L. A., and Rodman, T. (1967). American Heart fournal, 74, 710.

Stein, P. D., et al. (1967). American Heart fournal, 73, 730.

Wellman, H. N., et al. (1969), fournal of Nuclear Medicine, 10, 380

\section{Anaemia and Surgery}

\section{J. N. LUNN,* M.D., F.F.A. R.C.S. ; P. C. ELWOOD, † M.D.}

\begin{abstract}
Cummary: In 1,584 patients who had received an anaesthetic the association was examined between circulating haemoglobin level and the postoperative course (length of hospital stay, the occurrence of a complication, and death). In men a significant association $(P<0.05)$ was found for each index, but in women this was present only for death.
\end{abstract}

It is suggested that the hypothesis which best explains

\footnotetext{
* Senior Lecturer in Anaesthetics, Welsh National School of Medicine, Cardiff.

† Member of M.R.C. Epidemiology Research Unit, Cardiff.
}

\begin{abstract}
the associations, and is consistent with further analyses of the data, is that the preoperative haemoglobin level reflects the severity of the underlying condition which has necessitated surgery. A randomized clinical trial would test the alternative hypothesis that anaemia constitutes an additional risk in surgical procedures.
\end{abstract}

\section{Introduction}

Traditionally, anaemia is believed to increase the risk of surgery, and it is common practice for non-urgent surgery to be postponed if a patient is anaemic. The level of circulating 